\title{
Educação interprofissional em saúde e prática colaborativa: uma experiência na formação de residentes
}

\section{Interprofessional health education and collaborative practice: an experience in the formation of residents}

\author{
Amanda de Souza Nunes ${ }^{1}$, Elisabete Ferreira Mângia ${ }^{2}$, Helton Alves de Lima ${ }^{3}$ \\ http://dx.doi.org/10.11606/issn.2238-6149.v31i1-3p60-68
}

Nunes AS, Mângia EF, Lima HA. Educação interprofissional em saúde e prática colaborativa: uma experiência na formação de residentes. Rev Ter Ocup Univ São Paulo. 2020 jan.-dez.;31(1-3):60-8

RESUMO: A Educação Interprofissional em Saúde (EIP) e a prática colaborativa são estratégias recomendadas pela Organização Mundial da Saúde para a formação dos profissionais e qualificação das práticas em saúde. O Programa de Residência Multiprofissional em Saúde Mental com Ênfase em Dependência Química propõe um processo formativo alinhado á essas recomendações. Dentre os cenários formativos, destaca-se o Ambulatório Noturno de Cuidados em Álcool e outras Drogas (ANCAD). Este estudo visa compreender como a EIP e a prática colaborativa orientam o processo formativo no ANCAD; caracterizar a rotina de trabalho; descrever as percepções dos residentes; e identificar os conhecimentos e habilidades adquiridos no trabalho em equipe. De caráter qualitativo e exploratório, o estudo utilizou para coleta de dados a entrevista focal e observação direta. Os resultados indicaram que as atividades desenvolvidas são construídas e planejadas de maneira coletiva e compartilhada, fato que fomenta o desenvolvimento de competências para a interprofissionalidade. O ANCAD investe na criação de espaços de reflexão e debate sobre referenciais teóricos e atividades assistenciais pertinentes ao objetivo de qualificar os profissionais para o cuidado em saúde mental no campo de álcool e outras drogas.

PALAVRAS-CHAVE: Educação interprofissional em saúde; Prática colaborativa; Trabalho em equipe; Saúde mental; Álcool e outras drogas.
Nunes AS, Mângia EF, Lima HA. Interprofessional health education and collaborative practice: an experience in the formation of residentes. Rev Ter Ocup Univ São Paulo. 2020 Jan-Dec;31(1-3):60-8.

ABSTRACT: Interprofessional Health Education (IHE) and collaborative practice are strategies recommended by the World Health Organization (WHO) for the formation of professionals and qualification of health practices. The Multiprofessional Residency Program in Mental Health with Emphasis on Chemical Dependence proposes a forming process aligned with these recommendations. Among the learning scenarios, the Night Outpatient Clinic for Alcohol and Other Drugs (NOCAD) stands out. This study aims to understand how IHE and collaborative practice guide the formation process at NOCAD; characterize the work routine; describe resident's perceptions; and identify the knowledge and skills acquired in team work. Qualitative and exploratory, the study used focal interview and direct observation to collect data. The results indicated that the activities developed are built and planned in a collective and shared way, a fact that promotes the development of skills for interprofessionality. NOCAD invests in the creation of spaces for reflection and debate on theoretical references and assistance activities pertinent to the objective of qualifying professionals for mental health care in the field of alcohol and other drugs.

KEYWORDS: Interprofessional health education; Collaborative practice; Team work; Mental health; Alcohol and other drugs.

Artigo proveniente de trabalho de conclusão de residência apresentado ao Programa de Residência Multiprofissional em Saúde Mental com Ênfase em Dependência Química do Hospital das Clínicas da Faculdade Medicina da Universidade de São Paulo.

1. Terapeuta Ocupacional. Especialista em Saúde Mental com Ênfase e Dependência Química pelo Hospital das Clínicas da Faculdade de Medicina da Universidade de São Paulo - HC FMUSP. E-mail: amandasnunnes@gmail.com.

2. Prof ${ }^{\mathrm{a}} \operatorname{Dr}^{\mathrm{a}}$ do Departamento de Fisioterapia, Fonoaudiologia e Terapia Ocupacional da FMUSP. E-mail: mangeli@usp.br.

3. Psicólogo. Especialista em Saúde Mental, Álcool e outras Drogas e Mestre em Ciências da Saúde pela Faculdade de Medicina da Universidade de São Paulo - FMUSP. E-mail: lima.helton@gmail.com.

Endereço para correspondência: Amanda de Souza Nunes. Rua Almeida Brandão no 475 - Vila Guilhermina, São Paulo, SP. CEP: 03545-000 
Nunes AS, et al. Educação interprofissional em saúde e prática colaborativa. Rev Ter Ocup Univ São Paulo. 2020 jan.-dez.;31(1-3):60-8.

\section{INTRODUÇÃO}

A s transições demográfica e epidemiológica no incremento das condições crônicas e requerem o desenvolvimento de práticas que qualifiquem o cuidado e induzam ao trabalho em equipe com colaboração interprofissional ${ }^{1,2}$.

Neste contexto, a formação em saúde é desafiada a superar a atuação isolada e circunscrita ao âmbito de cada profissão e de seus conhecimentos específicos, o que representa um obstáculo ao desenvolvimento do trabalho em equipe na perspectiva da integralidade ${ }^{1}$. Nessa direção a Organização Mundial da Saúde (OMS) orienta a implementação da Educação Interprofissional em Saúde (EIP), definida como processo de aprendizagem que envolve a interação entre diferentes especialidades e que permite que os profissionais aprendam sobre os papéis, conhecimentos e competências dos demais, para efetivar a colaboração, melhorar os resultados, qualificar os processos de trabalho, otimizar e fortalecer os sistemas de saúde e orientar a atuação para as necessidades dos usuários ${ }^{1,3}$.

A EIP requer a participação ativa e o intercâmbio de saberes para a atuação integrada, reconhecimento da interdependência e colaboração interprofissional, redução da fragmentação e competição ${ }^{1,2}$. Tal estratégia sustenta a Prática Interprofissional em Saúde (PIP), que quando adotada nos serviços propicia a articulação e integração das intervenções, melhoria da resolutividade, qualidade da atenção, comunicação e a flexibilização dos papéis profissionais ${ }^{1}$.

O profissional de saúde colaborativo seria aquele que aprendeu e desenvolveu competências para trabalhar em equipe interprofissional, o que pode otimizar a utilização de recursos clínicos, a coordenação e acesso aos serviços e os resultados na saúde de usuários. Recomenda-se aos gestores de saúde a implementação de políticas de educação para favorecer e incentivar a EIP e a prática colaborativa ${ }^{3}$.

O desenvolvimento da EIP requer a formulação de programas de ensino direcionados a melhoria das competências dos estudantes para a articulação dos conhecimentos dos núcleos específicos ao campo de competências comuns da saúde $^{2}$. Para isso se propõe um conjunto de competências que visa contribuir na redefinição de diretrizes curriculares e viabilizar a aquisição de conhecimentos, habilidades, valores e atitudes fundamentais aos processos formativos direcionados a prática colaborativa:

comunicação interprofissional; cuidado centrado no paciente, família e comunidade; reconhecimento de papéis profissionais; entender os princípios da dinâmica do trabalho em equipe; liderança colaborativa e resolução de conflitos interprofissionais (p.7) ${ }^{2}$.

Essa modalidade de ensino requer a supervisão de preceptores das áreas profissionais envolvidas para contribuir com o reconhecimento mútuo dos papéis profissionais e na vivência do trabalho em equipe'. Esse ensino "envolve o debate de ideias, a definição de propósitos e objetivos, a delimitação da tarefa, tomada de decisão, assunção de responsabilidades e resolução de conflitos" (p. 6) ${ }^{2}$.

Alinhado a essa orientação, o Brasil adotou a Política Nacional de Educação Permanente em Saúde (EPS) visando o desenvolvimento da força de trabalho para o Sistema Único de Saúde (SUS) ${ }^{4}$. A incorporação desse referencial às práticas assistenciais e aos processos formativos levou o Ministério da Saúde (MS) em parceria com o Ministério da Educação (MEC) a criarem programas indutores de mudanças ${ }^{5}$.

Dentre esses programas, encontra-se a Residência Multiprofissional em Saúde (RMS). O investimento público na expansão das RMS relaciona-se a sua potencialidade política e pedagógica na formação de profissionais capacitados para a atuação condizente com os princípios do SUS e com o compromisso de modificação do modelo assistencial ${ }^{5}$.

\section{Ambulatório Noturno de Cuidados em Álcool e outras Drogas (ANCAD)}

Nos serviços comunitários de saúde mental a prática colaborativa proporcionada pela EIP pode:

aumentar a satisfação dos pacientes e dos profissionais, promover melhor aceitação do tratamento, reduzir a duração do tratamento, reduzir custos assistenciais, reduzir a incidências de suicídios, aumentar o tratamento para condições psiquiátricas e reduzir o número de consultas ambulatoriais" (p. 18) .

Orientada por esses princípios, a Rede de Atenção Psicossocial (RAPS) incorpora a "promoção de estratégias de educação permanente", e adota como um dos objetivos específicos a promoção de "mecanismos de formação permanente aos profissionais de saúde" .

Neste contexto apresentamos a experiência do Programa de Residência Multiprofissional em Saúde Mental com Ênfase em Dependência Química do Hospital das Clínicas da Faculdade de Medicina da Universidade de São Paulo (HC FMUSP). Este programa busca capacitar profissionais para o trabalho em equipe e promover processos formativos para qualificar o cuidado às pessoas 
com necessidades decorrentes do uso de crack, álcool e outras drogas ${ }^{7}$. Neste contexto, adota a perspectiva interdisciplinar e assume como eixo pedagógico a formação para o trabalho interprofissional colaborativo, visando:

desenvolver competências voltadas para as seguintes dimensões: comunicação, cuidado, papel profissional, funcionamento da equipe, liderança colaborativa, mediação de conflitos, gestão de Redes de Atenção à Saúde com ênfase na RAPS e educação permanente (p. 20) ${ }^{7}$.

O programa tem duração de 2 anos e oferece 8 vagas anuais, distribuídas nas áreas de enfermagem, psicologia, serviço social e terapia ocupacional. Os residentes desenvolvem atividades em serviços da RAPS do município de São Paulo: Unidade Básica de Saúde e Consultório na Rua, Centro de Atenção Psicossocial III Álcool e Outras Drogas e Hospital Especializado em Saúde Mental ${ }^{7}$. Na Atenção Hospitalar, atuam no Instituto de Psiquiatria (IPq) do HC FMUSP, na Enfermaria de Comportamentos Impulsivos (ECIM), Ambulatório do Grupo Interdisciplinar de Estudos de Álcool e Drogas (GREA) e no Ambulatório Noturno de Cuidados em Álcool e outras Drogas (ANCAD).

O ANCAD é um serviço ambulatorial implementado para responder à necessidade de ampliação do acesso de usuários e familiares ao cuidado em saúde mental, a construção de um espaço de experimentação de práticas assistenciais e de processos formativos baseados na aprendizagem em serviço. Iniciado em 2018, foi desenvolvido em parceria com o Programa de Residência Uniprofissional de Enfermagem em Psiquiatria e a Residência Médica de Psiquiatria, além da equipe de colaboradores da instituição.

Caracteriza o ANCAD o atendimento a demanda espontânea; integração entre profissionais de diferentes programas de residência; trabalho centrado na realização de grupos terapêuticos e de suporte sob a coordenação de residentes; atendimentos individuais e acolhimento de usuários; intervenções realizadas por no mínimo duas categorias profissionais; supervisão das atividades; e estudos temáticos com a presença de facilitadores, com vistas a mediar a EIP e fomentar a prática colaborativa.

As atividades do ambulatório ocorrem semanalmente às quartas-feiras, no período das 18:00 às 22:30 horas, assim distribuídas: reunião de equipe para a organização dos processos de trabalho; período de estudos e discussão de temáticas relativas ao trabalho; atendimento aos usuários e familiares por meio de acolhimento, coordenação de cuidado, atendimentos individuais, grupos de acolhimento, acompanhamento e de família; supervisão das práticas assistenciais.
Desse modo, este estudo tem o objetivo de compreender como a EIP e a prática colaborativa orientam o processo formativo dos residentes; caracterizar a rotina de trabalho; descrever as percepções dos residentes sobre o processo formativo e identificar os conhecimentos e habilidades adquiridos no e para o trabalho em equipe.

\section{METODOLOGIA}

Estudo de caso de caráter qualitativo e exploratório, voltado ao conhecimento da experiência e de seus resultados ${ }^{8}$. Os sujeitos da pesquisa foram os residentes inseridos no Programa de Residência Multiprofissional em Saúde Mental com Ênfase em Dependência Química, e a inclusão no estudo ocorreu a partir do interesse e disponibilidade. Os dados foram acessados a partir de entrevistas focais e observações direitas, sendo a coleta realizada no período de julho à outubro de 2019.

A entrevista focal foi dirigida a captar as opiniões dos residentes sobre o processo formativo, características da rotina do ANCAD e os conhecimentos e habilidades adquiridos no desenvolvimento do trabalho em equipe. Cada entrevista foi gravada e iniciada pela leitura do Termo de Consentimento Livre e Esclarecido (TCLE) e do roteiro de questões. Tiveram duração média de 40 minutos, sendo, posteriormente transcritas e analisadas. Participaram 4 residentes inseridos no programa e atuantes no ANCAD, sendo 1 assistente social, 1 enfermeiro, 1 psicólogo e 1 terapeuta ocupacional.

A observação direta da reunião de equipe e do período de estudos foi orientada por roteiro e realizada por dois residentes. Essa etapa também contemplou a análise de cronograma de estudo e respectivas temáticas escolhidas.

Para a análise adotou-se a análise de conteúdo temática. Os dados foram agrupados por semelhança de conteúdo, abrangendo as etapas de exploração prévia do material, seleção das unidades de análise e processo de categorização e subcategorização\%. Foram definidas três unidades de análise: rotina de trabalho, percepção dos residentes sobre o processo formativo e conhecimentos e habilidades adquiridos no trabalho em equipe. Os dados foram categorizados nas unidades de análises correspondentes para o estabelecimento de conexões com os referenciais teóricos adotados.

O estudo é parte do Projeto "Atenção Psicossocial em Saúde Mental - Álcool e drogas: desafios na construção de estratégias assistenciais e educacionais para o trabalho interprofissional" CAE 46268215.4.0000.0068, aprovado pelo Comitê de Ética do HC FMUSP. 
Nunes AS, et al. Educação interprofissional em saúde e prática colaborativa. Rev Ter Ocup Univ São Paulo. 2020 jan.-dez.;31(1-3):60-8.

\section{RESULTADOS}

No período de realização da pesquisa, participavam da equipe do ANCAD residentes, preceptores, supervisores e colaboradores das áreas de enfermagem, psicologia, psiquiatria, serviço social e terapia ocupacional, perfazendo 14 membros. Os residentes integram a equipe do ambulatório por período determinado de acordo com seu processo formativo o que gera rotatividade periódica dos participantes.

\section{Rotina de trabalho}

A equipe do ANCAD estrutura a rotina de trabalho e define sua organização, divisão de tarefas e responsabilidades. Para os entrevistados, esse processo é resultado das atividades compartilhadas nas reuniões de equipe, períodos de estudos e supervisões. As práticas assistenciais são realizadas de forma compartilhada e não estão restritos aos núcleos profissionais.

Para os participantes da pesquisa, a reunião de equipe se caracteriza como momento de discussão da rotina de trabalho, espaço de coletivização de reflexões e também de decisões administrativas.

A observação direta atestou que a reunião se constitui como espaço de estímulo ao processo formativo e ao trabalho em equipe. Nela são compartilhadas decisões acerca do trabalho, da assistência e das temáticas para estudo. Observou-se que há interação horizontal entre os membros da equipe com partilha de opiniões e percepções, além da divisão das tarefas de acolhimentos, atendimentos individuais e grupais.

Os períodos de estudo consistem na seleção e discussão de temáticas para aprofundamento teórico e instrucional e objetivam aprimorar a atuação a partir das necessidades identificadas na prática.

"Os temas são escolhidos a partir da necessidade de aprofundar algo que surgiu no ambulatório (...). Por exemplo, estudamos sobre violência e a Lei Maria da Penha, e como abordar casos de violência no ambulatório”. (E.3)

A articulação entre teoria e prática promove o processo coletivo de reflexão. Cada profissional realiza sua pesquisa e a compartilha com a equipe. Todavia, identificaram-se dificuldades relacionadas à execução do planejamento dos estudos, devido à emergência de outras questões, como a entrada de novos profissionais no ambulatório, fato que demanda a recepção e discussão sobre a divisão de atribuições e práticas assistenciais.

Na supervisão discutem-se os casos acompanhados, práticas assistenciais e atendimentos individuais.
Os entrevistados referem que há flexibilidade em relação aos conteúdos discutidos, como manejos grupais e condutas profissionais, além da abordagem de situações críticas vivenciadas no curso do trabalho. Consideram que esta atividade fomenta o processo de aprendizagem em equipe e contribui para qualificar o cuidado a partir do diálogo com referenciais teóricos e conhecimentos de diferentes núcleos de saberes.

A equipe conta com 3 supervisores (terapeuta ocupacional, assistente social e médico psiquiatra) que realizam a mediação do processo de reflexão da atuação profissional e do trabalho em equipe. Há estímulo ao compartilhamento de olhares e perspectivas entre os participantes.

"Nós vamos trazendo elementos dos casos, os supervisores e até mesmo os outros profissionais - que possuem outra visão-contribuem com alguma perspectiva teórica”. (E.3)

A supervisão estimula a troca de conhecimentos específicos e a interação entre os profissionais. O reconhecimento de que há diferentes possibilidades de avaliar e abordar determinada problemática é valorizado no trabalho em equipe.

"No ambiente de supervisão, a interação ocorre de forma intensa, pois trazemos as contribuições de cada profissão, por exemplo, como a psicologia ou a terapia ocupacional a dinâmica grupal”. (E.4)

\section{Práticas assistenciais}

As práticas grupais, coordenadas por residentes e supervisionadas por preceptores, são as principais ofertas de cuidado do ambulatório. Caracterizam-se pela interação e compartilhamento de responsabilidades, situação que é compreendida como estímulo à articulação de saberes, ao trabalho em equipe e cuidado qualificado.

\footnotetext{
"Os grupos são coordenados por dois profissionais de diferentes áreas. Nos atendimentos individuais também há essa divisão. Acredito que vamos criando uma linguagem em comum sem abdicar necessariamente da nossa linguagem especifica, fruto da nossa formação”. (E.4)
}

A coordenação de cuidado - que contempla os atendimentos individuais - é definida pelos entrevistados como a possibilidade de acompanhar os casos de maneira singular, com vistas a acessar a história de vida dos sujeitos, seus itinerários terapêuticos, demandas e necessidades. 
Em relação aos atendimentos específicos de categorias profissionais, os entrevistados afirmam que este tipo de intervenção ocorre em ocasiões esporádicas e de acordo com as necessidades dos usuários.

\section{"Não é a característica do ambulatório pensar nas especificidades das profissões. Pensamos a partir do trabalho em equipe, como nós podemos dividir as funções e as tarefas". (E.2)}

As práticas assistenciais são planejadas e executadas coletivamente. As especificidades profissionais são acessadas nas discussões e reflexões sobre o trabalho. Ademais, nas reuniões, estudos e supervisões são organizadas as práticas assistenciais e discutidos os referenciais teóricos adotados.

No ANCAD também ocorre à oferta de grupos temáticos que agregam usuários e familiares para que dialoguem acerca de temas específicos, como por exemplo, as situações de violência contra as mulheres e a Luta Antimanicomial.

A articulação da rede setorial e intersetorial, e o trabalho em rede foram considerados como práticas assistenciais, visto que uma das premissas do ambulatório é que os usuários acessem os cuidados em saúde nos serviços da sua comunidade e de acordo com suas necessidades.

\section{Processo formativo}

Nesse cenário, o processo formativo visa à construção compartilhada entre as categoriais profissionais. A interação entre os saberes agrega conhecimentos teóricos e práticos ao repertório dos membros da equipe. São estabelecidas atividades para potencializar o processo formativo, considerando a particularidade da equipe. A articulação entre diferentes núcleos de saberes permeia as interações da equipe, bem como a validação das opiniões e perspectivas de cuidado, aspecto que é considerado fundamental, pois dialogam com a complexidade das necessidades usuários e com o caráter multifatorial do consumo de álcool e outras drogas.

"É comprovado que o cuidado em álcool e outras drogas não está restrito a uma categoria, como médico e psicólogo, mas sim de várias categorias juntas que se articulam e olham para o individuo de uma forma mais completa". (E.1)

Neste contexto, a aprendizagem no ambulatório ocorre pela prática profissional. Os entrevistados percebem a aprendizagem em serviço como base da formação.

"O processo de aprendizagem ocorre a partir da prática, é assim que estudamos ou refletimos". (E.2)
Aprendizados prévios são mobilizados a interagir com as situações encontradas na prática, o que fomenta a interação e a articulação dos saberes. Os entrevistados afirmam que essa dinâmica possibilita a construção de recursos para lidarem com as dificuldades presentes na rotina de trabalho.

"Esse serviço tem um aspecto muito interessante que éo de utilizar questões que surgem no ambiente prático para reflexão, e formalizá-las em objeto de estudos". (E.4)

Os entrevistados mencionam a prática da escuta, definida como a habilidade de estabelecer comunicação efetiva entre os membros da equipe, como importante componente da aprendizagem dialógica e como principal ferramenta de trabalho no ambulatório.

"Eu aprendi a ouvir mais as outras pessoas (...). Às vezes nós tomamos uma decisão conforme estamos habituados, pela especificidade da profissão e daquele momento, porém precisamos estar abertos para escutar o outro". (E.2)

Segundo os entrevistados o ambulatório propicia uma nova forma de se relacionar e de desenvolver a prática profissional que possui como demarcador as relações e interações coletivas no âmbito do trabalho, suas especificidades e benefícios.

"O ANCAD é um lugar que estou aprendendo a pensar junto, compartilhar e a construir processos de cuidado que não são resultado apenas da minha percepção, e sim do trabalho em equipe". (E.2)

Outros aprendizados são relatados, como o manejo de grupos e a aproximação com a rede setorial da saúde. As experiências prévias dos residentes nos campos de estágio da atenção básica, psicossocial e hospitalar - como consta no programa da residência - além dos conhecimentos mobilizados nas reuniões, estudos e supervisões, são reconhecidos como potencializadores dos resultados da aprendizagem em serviço.

\section{DISCUSSÃO}

\section{Trabalho em equipe com características interprofissionais e colaborativas}

A reflexão proposta está relacionada ao modelo de trabalho desenvolvido no ANCAD, como lócus formativo e assistencial baseado no trabalho em equipe dos residentes. 
Nunes AS, et al. Educação interprofissional em saúde e prática colaborativa. Rev Ter Ocup Univ São Paulo. 2020 jan.-dez.;31(1-3):60-8.

O trabalho em equipe corresponde ao trabalho coletivo que se estrutura na relação recíproca entre as intervenções técnicas e interação entre os profissionais, se coloca como estratégia para a integração das especialidades e profissões e base para o desenvolvimento do cuidado integral, centrado nas necessidades dos sujeitos ${ }^{1,2}$. Os resultados apresentados atestam que o ANCAD assume essas premissas e mostra que, nele o trabalho em equipe assume características interprofissionais, na medida em que a EIP é adotada como norteadora do processo formativo dos residentes. As características da interprofissionalidade observadas potencializam o trabalho com destaque para: a tomada de decisões compartilhadas, o reconhecimento do trabalho dos demais profissionais, a interdependência das ações e a flexibilidade da divisão do trabalho e das fronteiras entre as especialidades ${ }^{2}$.

Os entrevistados mostram que as tomadas de decisões compartilhadas são desenvolvidas nas reuniões de equipe e na construção das práticas assistências. Questões relativas à rotina do serviço e ao projeto assistencial são discutidas coletivamente e o reconhecimento do trabalho dos demais profissionais é evidenciado na identificação de competências e conhecimentos específicos, assim como a colaboração dos diferentes saberes no desenvolvimento das atividades.

Os entrevistados destacaram a interdependência das ações na construção do cuidado e nas decisões relativas ao funcionamento do ambulatório. Os saberes das diferentes categoriais profissionais são vistos como complementares e sua articulação são indispensáveis para o cuidado no campo da saúde mental, álcool e outras drogas.

A flexibilidade na divisão do trabalho e no estabelecimento de fronteiras entre as especialidades se evidencia no planejamento e proposições assistenciais. Apesar disso, os saberes das especialidades são reconhecidos e requisitados nas reuniões, supervisões e reflexões.

A rotina do ANCAD é centrada no compartilhamento de atribuições, práticas assistências e atividades entre os profissionais, fato que singulariza o trabalho em equipe. O trabalho se organiza a partir da percepção da equipe acerca das necessidades de saúde dos usuários, conjuntura que de acordo com Peduzzi et al. ${ }^{10}$ possibilita o estabelecimento de conexões com a prática colaborativa que ocorre quando os membros da equipe procuram trabalhar juntos em favor da qualificação e integralidade do cuidado. Na prática colaborativa as necessidades de saúde são consideradas como eixo norteador das práticas assistenciais, cabendo aos sistemas e equipes de saúde o desenvolvimento de projetos e ações que visem a construção do cuidado integral em conjunto com os usuários e sociedade ${ }^{3}$.

No campo da saúde mental e álcool e outras drogas, a diretriz da atenção integral é incorporada no sentido de qualificar as respostas às condições de vida e saúde das pessoas e, para tanto, é necessário formar recursos humanos para lidar com a complexidade dessas questões ${ }^{11}$. Notou-se que o ANCAD adotou tais norteadores no desenvolvimento dos processos de trabalho. No âmbito do trabalho em equipe interprofissional, tais objetivos formativos e assistenciais são potencializados nos processos de supervisão e reunião de equipe ${ }^{2}$.

Diferentemente da concepção tradicional na qual a supervisão se caracteriza pela interlocução de uma equipe com um supervisor externo ${ }^{2}$, no ambulatório os supervisores auxiliam a equipe no processo de reflexão e avaliação do trabalho e se colocam como facilitadores do processo formativo dos residentes, compartilhando seus conhecimentos específicos. As reuniões de equipe, propiciam a real integração e colaboração entre os membros e são direcionadas aos resultados esperados para o cuidado aos usuários ${ }^{2}$.

Outro aspecto importante é a articulação entre núcleos de saberes. Os entrevistados destacaram a interação entre as diferentes categoriais profissionais na qual as especificidades e experiências de cada membro da equipe são utilizadas para a construção das intervenções. Essa característica pode ser problematizada a partir dos conceitos de núcleos e campos de saberes. O núcleo corresponde a aglutinação de determinados saberes e práticas, demarcando uma identidade profissional; e o campo representa o espaço de fronteiras permeáveis e limites imprecisos na qual diferentes disciplinas buscam respaldo em outras para efetivar o seu trabalho ${ }^{12}$.

Neste estudo, as articulações entre os núcleos de saberes da psicologia, enfermagem, terapia ocupacional e serviço social são percebidas pelos entrevistados como modo de atender as necessidades dos usuários, levando em conta a complexidade envolvida no consumo de álcool e outras drogas.

Em conjunto aos núcleos de saberes, encontram-se os conhecimentos que respaldam a atuação no ANCAD, provenientes dos campos de saberes da saúde mental, das políticas de saúde e da EIP, entretanto não são evidentes as fronteiras existentes entre esses saberes. Núcleo e campo são mutantes e se interinfluenciariam, tornando-se impossível identificar limites exatos entre esses ${ }^{12}$.

\section{Aprendizagem em serviço: conhecimentos, habilidades e atitudes para o trabalho em equipe}

O modelo de trabalho no ANCAD concentra-se na identificação da aprendizagem como mediação entre prática profissional e os resultados esperados para a formação em saúde. A aprendizagem em serviço é percebida pelos entrevistados como eixo central da formação, a partir da prática profissional e do trabalho em equipe, o que dialoga 
com a Política de Educação Permanente em Saúde ${ }^{13}$ incorporada no projeto político pedagógico da residência. Diante disso, a reflexão da prática:

confere centralidade ao residente no processo de ensinoaprendizagem, estimulando o pensamento crítico, o agir ético, o protagonismo, a autonomia e a co-responsabilização na construção do saber (p. 21)

Para o desenvolvimento dessa competência, os residentes assumem atitudes de protagonismo e autonomia para propor temáticas de estudos e partilham com a equipe a tarefa de qualificar os processos de trabalho e de cuidado. Outra característica desse processo é a aquisição de habilidades de comunicação e mediação de conflitos, necessárias para a integração da equipe e para o trabalho interprofissional colaborativo.

A comunicação entre os membros da equipe é central para o desenvolvimento do trabalho e articulação das ações, pois a interação entre os profissionais depende da mediação simbólica da linguagem ${ }^{10}$. A ruptura do diálogo, a utilização de linguagens herméticas e a impossibilidade de trocas entre os profissionais dificultam a operacionalização do trabalho em equipe interprofissional ${ }^{2}$.

Para tanto, torna-se necessário incluir, na rotina dos serviços, recursos para o aprimoramento da comunicação ${ }^{10}$. No ANCAD, a reunião de equipe, o período de estudos e a supervisão são espaços privilegiados para exercer essa habilidade e promover à participação dos membros, propiciando o estímulo à capacidade de argumentação e ao pensamento crítico.

A mediação dos conflitos que surgem da interação constante entre profissionais, que possuem formações e experiências diversas, é outra habilidade central a ser desenvolvida. A negligência de situações de conflitos pode ocasionar barreiras para a prática colaborativa, visto que são frutos de divergências de percepções e ideias que colocam os membros da equipe em posições antagônicas, e que tem como causas mais frequentes os "problemas de comunicação, estrutura organizacional, disputa de papéis, escassez de recursos, mal-entendidos, falta de compromisso profissional e outras" (p. 804) $)^{14}$.

A medicação de conflitos é vista como uma abordagem promotora de modificações organizacionais, grupais e profissionais que potencializam a inovação e produtividade ${ }^{14}$. No projeto político pedagógico da residência, a mediação é identificada como competência que:

compreende a capacidade de abordar de forma positiva e construtiva os conflitos e divergências surgidas no contexto do cuidado. Habilidades para encaminhar a resolução de conflitos, e reconhecer a possibilidade de encontrar soluções construtivas para superar as divergências (p. 20) ${ }^{7}$.

Os entrevistados citaram como dificuldades, a conciliação de diferentes ritmos para o trabalho e a construção de processos de cuidado compartilhados. É comum o surgimento de divergências acerca do trabalho, entretanto espera-se que os residentes desenvolvam essa competência. Ao sugerirem, de maneira implícita, a mediação de conflitos como habilidade adquirida no ANCAD, é possível afirmar que os elementos relacionais são vistos como relevantes para os processos de trabalho, fato que pode instrumentalizar os residentes para prática colaborativa em outros contextos de atenção.

Em relação às percepções dos residentes sobre os conhecimentos adquiridos no trabalho em equipe, encontrase o desenvolvimento de práticas grupais no campo da saúde mental com ênfase em álcool e outras drogas. Em diálogo, o conteúdo do programa de residência inclui a aproximação e reflexão sobre diferentes modalidades grupais, como grupos psicoeducativos e terapêuticos ${ }^{7}$. Em saúde mental, os grupos são compreendidos como tecnologia de cuidado complexa e diversificada que possibilita trocas de experiências e transformações subjetivas ${ }^{15}$. Nesse sentido, a inserção no ANCAD induz o aprendizado sobre as teorias grupais a partir de vivências práticas compartilhadas com distintas categoriais profissionais que detém variados arcabouços teóricos.

Outro conhecimento citado pelos participantes referese às trocas de experiências oriundas dos distintos campos de prática da residência, que permite a aproximação com os demais pontos de atenção e componentes da RAPS, fato que pode viabilizar a continuidade e integralidade do cuidado. Em consonância a esse cenário, a RAPS assume como um dos seus objetivos gerais a garantia da "articulação e integração dos pontos de atenção das redes de saúde no território, qualificando o cuidado por meio do acolhimento, do acompanhamento contínuo e da atenção às urgências"6. Ademais, as vivências dos residentes em diferentes pontos de atenção da RAPS reverberam no trabalho realizado no ANCAD, visto que permite o contato com outras realidades e estratégias de intervenção, enriquecendo o processo formativo e o cuidado em saúde.

\section{CONSIDERAÇÕES FINAIS}

Esta pesquisa visou relatar a experiência do processo formativo de residentes no ANCAD, com o objetivo de compreender como a EIP e a prática colaborativa oferecem 
Nunes AS, et al. Educação interprofissional em saúde e prática colaborativa. Rev Ter Ocup Univ São Paulo. 2020 jan.-dez.;31(1-3):60-8.

subsídios para o desenvolvimento do trabalho em equipe, das praticas assistenciais e atividades no serviço.

Neste contexto, evidenciou-se o compartilhamento das atividades entre diferentes categoriais profissionais, o trabalho em equipe com características interprofissionais e o potencial para a prática colaborativa. Logo, a inserção no ANCAD permite a continuidade do processo formativo em nível de especialização privilegiando o aprendizado interativo entre as categoriais profissionais.

Os resultados convergiram com os princípios e diretrizes da EIP, da prática colaborativa e elementos norteadores descritos no programa de residência, circunstância que indica um posicionamento político e pedagógico na estruturação do ambulatório e no processo formativo em saúde mental e no campo de álcool e outras drogas.

Ademais, a inserção no ambulatório propiciou aos residentes aproximações com as habilidades de comunicação e medicação de conflitos, conhecimentos relativos às práticas grupais e a RAPS.

No decorrer da pesquisa, foram mencionados aspectos que podem fragilizar a proposta do ANCAD, como a dificuldade de manutenção do período de estudos e a rotatividade de residentes.

Não sem inúmeros desafios, os resultados indicam a construção de um aprendizado em que ocorre a articulação entre os conhecimentos e competências dos profissionais - com a manutenção da especificidade - e de um saber coletivo que é citado como singularidade do trabalho realizado no ambulatório.

Por fim, essa experiência descrita em estágio inicial de implementação indica o alinhamento teórico da equipe acerca do que se deseja produzir enquanto modelo de cuidado, situação que indica a importância da criação de espaços que fomentem novas experiências no que tange os processos formativos em saúde mental.

Participação dos autores: Amanda de Souza Nunes: Aluna responsável pelo trabalho de conclusão de curso de residência multiprofissional, que originou o presente manuscrito. Participou de todas as etapas de formulação do projeto de pesquisa e do manuscrito. Elisabete Ferreira Mângia: Orientadora do projeto de pesquisa. Participou da formulação de todas as sessões do presente manuscrito, incluindo sua revisão final. Helton Alves de Lima: Participou de todas as etapas do processo de orientação e revisão do projeto de pesquisa.

\section{REFERÊNCIAS}

1. Peduzzi M, Norman IJ, Germani ACCG, Silva JAM, Souza GC. Educação Interprofissional: formação de profissionais de saúde para o trabalho em equipe com foco nos usuários. Rev Esc Enferm USP. 2013;47(4):977-83. https://doi.org/10.1590/S0080-623420130000400029.

2. Peduzzi M, Oliveira MAC, Silva JAM, Agreli HLF, Miranda Neto MV. Trabalho em equipe, prática e educação interprofissional. In: Martins MA, et al. Clínica médica: atuação da clínica médica, sinais e sintomas de natureza sistêmica, medicina preventiva, saúde da mulher, envelhecimento e geriatria. São Paulo: Manole; 2016. v.1, Cap.17, p.1-9. Disponível em: https://edisciplinas.usp.br/ pluginfile.php/3011330/mod_resource/content $/ 1 /$ Trabalho $\% 20$ em\%20equipe.pdf.

3. Organização Mundial da Saúde (OMS). Gabinete da Rede de Profissões de Saúde. Enfermagem \& Obstetrícia do Departamento de Recursos Humanos para a Saúde. Marco para ação em educação interprofissional e prática colaborativa. Gabinete da Rede de Profissões. Geneva; 2010. Disponível em: https://www.paho.org/bra/images/stories/documentos/ marco_para_acao.pdf.

4. Brasil. Ministério da Saúde. Portaria $n^{\circ} 198,13$ de fevereiro de 2004. Brasília: Ministério da Saúde; 2004.
5. Brasil. Ministério da Saúde. Secretaria de Gestão do Trabalho e da Educação na Saúde. Departamento de Gestão da Educação na Saúde. Residência multiprofissional em saúde: experiências, avanços e desafios. Brasília: Ministério da Saúde; 2006. Disponível em: http://bvsms.saude.gov.br/ bvs/publicacoes/residencia_multiprofissional.pdf.

6. Brasil. Ministério da Saúde. Portaria n ${ }^{\circ} 3.088$, de 23 de dezembro de 2011. Brasília: Ministério da Saúde; 2011. Disponível em: http://bvsms.saude.gov.br/bvs/saudelegis/ gm/2011/prt3088_23_12_2011_rep.html.

7. Universidade de São Paulo - USP. Projeto Político Pedagógico da Residência Multiprofissional em Saúde Mental com Ênfase em Dependência química. São Paulo; 2018.

8. Yin RK. Estudo de caso: planejamento e métodos. 2a ed. Porto Alegre. Ed. Bookman; 2001.

9. Campos CJG. Método de análise de conteúdo: ferramenta para a análise de dados qualitativos no campo da saúde. Rev Bras Enferm. 2004;57(5):611-4. https://doi.org/10.1590/ S0034-71672004000500019.

10. Peduzzi $\mathrm{M}$, et al. Trabalho em equipe na perspectiva da gerência de serviços de saúde: instrumentos para a construção da prática interprofissional. Physis Rev Saúde 
Coletiva (Rio de Janeiro). 2011;21(2):629-46. https:// doi.org/10.1590/S0103-73312011000200015.

11. Brasil. Ministério da Saúde. Secretaria Executiva Coordenação Nacional de DST e AIDS. A Política do Ministério da Saúde para a Atenção Integral a Usuários de Álcool e outras Drogas. Brasília: Ministério da Saúde; 2003. (Série B. Textos Básicos de Saúde). Disponível em: http://bvsms.saude.gov.br/bvs/ publicacoes/pns_alcool_drogas.pdf.

12. Campos GWS. Saúde pública e saúde coletiva: campo e núcleo de saberes e práticas. Rev Cien Saúde Coletiva. 2000;5(2):21930. https://doi.org/10.1590/S1413-81232000000200002.

13. Brasil. Ministério da Saúde. Portaria $\mathrm{n}^{\circ} 278$, de 27 de fevereiro de 2014. Brasília: Ministério da Saúde; 2014. Disponível em: http://bvsms.saude.gov.br/bvs/saudelegis/gm/2014/ prt0278_27_02_2014.html

14. Spagnol CA, Santiago GR, Campos BMO, Badaró MTM, Vieira JS, Silveira APO. Situações de conflitos vivenciadas no contexto hospitalar: a visão dos técnicos e auxiliares de enfermagem. Rev Esc Enferm USP. 2010;44(3):803-11. https://doi.org/10.1590/S0080-62342010000300036

15. Brasil. Ministério da Saúde. Secretária de Atenção à Saúde. Departamento de Atenção Básica. Departamento de Ações Programáticas Estratégicas. Saúde mental. Departamento de Ações Programáticas Estratégicas. Brasília: Ministério da Saúde; 2013. (Caderno de Atenção Básica, n 34). Disponível em: http://bvsms.saude.gov.br/bvs/publicacoes/cadernos atencao_basica_34_saude_mental.pdf. 